\title{
Inscribing the blank slate: the growing role of modified alginates in tissue engineering
}

\section{Editorial}

The recent and steady upsurge in the use of alginate in tissue engineering has been well documented over the past decade. Due to the highly tailorable properties of the brown-algae derived polysaccharide, numerous researchers have employed it as a biomaterial for use in applications as widely varying as the polymer itself. Alginate fibers are a well-known wound dressing technique, nanodrops and microbeads of alginate can be used for specialized drug delivery, macroblocks of alginate are being 3D printed to create large-scale tissue reconstructions, and in situ crosslinked alginate can be injected into areas of need with minimal damage to surrounding tissues. $^{1-5}$

Commercially available alginate is derived from brown algae that has been extracted and purified into an acid (alginic acid) or a salt (Naalginate). The purified polysaccharide contains varying compositions and blocks of $\beta$-D-mannuronic (M) and $\alpha$-L-guluronic $(\mathrm{G})$ acid chains, the ratios typically dependent on the brown algae source and the level of processing. Bacterial biosynthesis, although costly, can also provide more tightly-controlled chemical configuration to tailor a higher concentration of either acid for varying gel characteristics; high mannuronic content leads to a higher elasticity, while high guluronic content gels exhibit an enhanced stability and mechanical strength. ${ }^{6}$

Alginate has been used commercially in multiple applications; in the food industry alginate has been a popular stabilizer for emulsions such as ice cream, jelly, toothpaste and paint. Alginate has been used in wastewater treatment systems and ethanol production. Clinically, alginate has commonly been used as a dental impression material, an adhesive or sustained-release agent for medicinal tablets, and as a hemostatic and absorbent wound dressing. ${ }^{6}$ Within the tissue engineering industry, alginate matrices have been employed in cell culture since 1980 as an encapsulation agent for cells. ${ }^{7}$ Since then, alginate has been extensively characterized, and discovered to be biocompatible, non-toxic, biodegradable, and non-immunogenic. ${ }^{8}$

The properties of alginate make it unique in the sense that it appears to have very few characteristic limitations. Alginate is easily crosslinked naturally and reversibly by use of a divalent cation within the guluronic chains; most often calcium is employed over the stronger bonding, but less biocompatible strontium or barium. Varying the accessibility to these cations can alter the gel characteristics significantly: higher concentrations of alginate bonds, with a higher ion concentration and/or a higher guluronic acid content, invariably strengthen the ultimate mechanical properties of the gel. However, rapid ionic crosslinking can cause inhomogeneity, mesh tightening and lack of elasticity. ${ }^{9}$ A balance between gel strength and homogeneity must be sought to maintain proper viscoelasticity and to prevent improper shape alterations. Alternatively, alginate can also be crosslinked by a $\mathrm{pH}$ reduction below the uronic acid $\mathrm{pKa}$ values; the solution viscosity increases with a decreasing $\mathrm{pH}$ as the carboxylate groups protonate and form hydrogen bonds. Covalent crosslinking has been shown to increase elasticity and create more stable and robust networks as the bonds lack the ability to dissociate and reform as in the case of ionic gels. ${ }^{10}$
Volume I Issue 2 - 2016

\author{
Emily A Growney Kalaf, Scott A Sell \\ Department of Biomedical Engineering, Saint Louis University, \\ USA
}

Correspondence: Scott A Sell, Department of Biomedical Engineering, Parks College, Saint Louis University, USA, Email ssell@slu.edu

Received: September 30, 2016 | Published: November 08, 2016

In an effort to further increase alginate capabilities and combat issues regarding a lack of inherent cell-binding sites, researchers are modifying the base polymer to attach side groups such as basement membrane proteins or methacrylate groups to add strength or a different crosslinking method to the alginate. Attachment of cellular adhesion motifs has typically been achieved through carbodiimide chemistry, and other modification techniques such as partial periodate oxidation, acetylation, and amidation have been applied successfully. The backbone of the alginate polymer contains multiple free hydroxyl and carboxyl groups, which allow for ease of modification of solubility, physicochemistry, hydrophilicity, and biological characteristics. ${ }^{10,11}$

Before modifying your alginate, it is necessary to determine what parameters you would like to change. Since the properties of these alginates are so tailorable, coming up with a design strategy to determine which method you would employ is desirable. Oxidation of the hydroxyl groups are used for drug delivery to increase the biodegradability of the alginate chains and decrease the stiffness of the polymer. Reductive amination of this oxidized alginate can endow amphiphilic properties such as heavy metal adsorption and lower surface tension. Sulfating alginate shows high blood compatibility and anticoagulant activity as it creates a structure similar to that of heparin sulfate. Copolymerization lends properties such as super absorbency (alginate + sodium acrylate), flocculation (acrylamide + alginate, alginate-g-vinyl sulfonic acid), and resistance to biodegradation. Modification of the carboxyl groups by esterification attaches alkyl groups to increase the hydrophobicity and amphiphilicity of alginate, allowing encapsulation of molecules of any phobicity while retaining the native alginate properties. Alginates are insoluble in organic solvents in their natural form; acylation of alginates allows for homogenous reaction in organic solvents and long alkyl chains can then be esterified onto the backbone. Amphiphilic alginates are highly useful as drug delivery systems; they can also exhibit long term stability and show prolonged drug release. ${ }^{12}$

In the field of tissue engineering, a very common modification of alginate is the introduction of bioactive ligands. The addition of these peptides, typically through their additions as side chains coupled to the carboxyl group via carbodiimide chemistry, provide the inherent cell-binding sites which alginate inherently lacks. As alginate gels 
are increasingly utilized in cell culture studies, these gels, which can be tailored specifically for varying stiffness and other mechanical properties in both $2 \mathrm{D}$ and $3 \mathrm{D}$ culture, are very often functionalized to maximize the effectiveness of the alginate in culture. Cells in 3D culture can migrate out of alginate gels, dependent on the porosity as well as the attached binding sites. Cellularized alginates, particularly alginate functionalized with cell adhesion ligands, have been employed successfully in angiogenesis when coupled with VEGF in mice. Functionalized alginate has been researched as a way to support pancreatic islet cells, achieve neurite outgrowth, and significantly increase repopulation and regeneration of damaged muscle tissue. Due in part to the ability to fill defects by creating homogenous geometries with internal ionic gelation, the ease of manipulation and functionalization of alginate makes its use advantageous over other materials in the field of cartilage and bone regeneration. ${ }^{8,9}$

Commercially, very few modified alginates are available. To avoid exorbitant costs of purchasing pre-modified polymers, many published protocols with relatively simple chemistry exist to functionalize alginate in your own labs. In the near future it is estimated by the authors that due to the relative ease of manufacture, highly tailorable mechanical, chemical and biological properties, alginate will be even more readily used in a modified form by researchers in tissue engineering and many other fields of interest. Alginate hydrogels suffer the limitations of all hydrogels in that the mechanical properties are weaker than other biomaterials; however, the wide range of uronic acid ratios, cross linking strategies and modifications to the alginate chain allow it to be finely tailored to the application. This will allow the alginates to play a more active role in wound healing, drug delivery, and in vitro applications. Alginate, known as the 'blank slate' of natural polymers, may soon enable significant advances in biomedicine.

\section{Acknowledgements}

None.

\section{Conflict of interest}

The author declares no conflict of interest.

\section{References}

1. Sell SA, Ericksen JJ, Reis TW, et al. A case report on the use of sustained release platelet-rich plasma for the treatment of chronic pressure ulcers. $J$ Spinal Cord Med. 2011;34(1):122-127.

2. Hinton TJ, Jallerat Q, Palchesko RN, et al. Three-dimensional printing of complex biological structures by freeform reversible embedding of suspended hydrogels. Sci Adv. 2015;1(9):e1500758.

3. Powers JG, Higham C, Broussard K, et al. Wound healing and treating wounds: Chronic wound care and management. J Am Acad Dermatol. 2016;74(4):607-625.

4. Tønnesen HH, Karlsen J. Alginate in drug delivery systems. Drug Dev Ind Pharm. 2002;28(6):621-630.

5. Bogyu Choi, Xian Jun Loh, Aloysius Tan, et al. Introduction to In Situ Forming Hydrogels for Biomedical Applications. In In-Situ Gelling Polymers. Springer. 2014;5-35.

6. Goh CH, Heng PWS, Chan LW. Alginates as a useful natural polymer for microencapsulation and therapeutic applications. Carbohydrate Polymers. 2012;88(1):1-12.

7. Lim F, Sun A. Microencapsulated islets as bioartificial endocrine pancreas. Science. 1980;210(4472):908-910.

8. Klöck G, Pfeffermann A, Ryser C, et al. Biocompatibility of mannuronic acid-rich alginates. Biomaterials.1997;18(10):707-713.

9. Growney Kalaf EA, Flores R, Bledsoe JG, et al. Characterization of slow-gelling alginate hydrogels for intervertebral disc tissue-engineering applications. Mater Sci Eng C Mater Biol Appl. 2016;63:198-210.

10. Lee KY, Mooney DJ. Alginate: properties and biomedical applications. Prog Polym Sci. 2012;37(1):106-126.

11. Pawar SN, Edgar KJ. Alginate derivatization: a review of chemistry, properties and applications. Biomaterials. 2012;33(11):3279-3305.

12. Ji Sheng Yang, Ying Jian Xie, Wen He. Research progress on chemical modification of alginate: A review. Carbohydrate Polymers. 2011;84(1):33-39. 